Symbols.com. Access: http://www.

symbols.com.

Symbols.com is the Internet version of Thought Signs (I.O.S. Press, 1994) by Carl Liungman. The book was well received in its original form Dictionary of Symbols (ABC-Clio. 1991), and the online version should be as well. The site has entries for 2,500 non-pictorial ideograms spanning the history of Western civilization.

The types of symbols used are vast and can be categorized only with difficulty into broad groups: cultural, "sub"-cultural, historic,

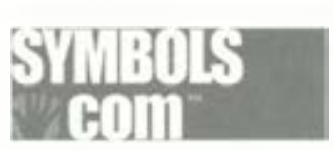
scientific, mathematical, and religious. Some examples include: Celtic, Gypsy,

hobo, and hippie signs; cartographic and musical notations; astronomical and chemistry ideograms; and mystic and prehistoric symbols. Coverage varies in length, but entries include a definition for each distinct use of the image and hyperlinks to related images. Occasionally there will be a notation of where and how the symbol appears in non-Western use.

Because there are graphic characteristics that typify symbols, access to the contents is through a word index and a graphic index. The word index is a straightforward search for a symbol that has a particular meaning. This is accomplished through an alphalbetical and numeric list of terms. The graphic index will retrieve groups of symbols based on their graphic characteristics, rather than meaning. There are 54 groups in all. Users may choose from a variety of pull-down menu options or from the "advanced graphic search" grid.

The graphic nature of the content is well suited for the Internet medium, and the site certainly fills a void in Internet reference collections. Unfortunately, there is no bibliography for the work or individual entries, and some of the names of symbols were not translated from the Swedish (e.g. "om" is spelled "aum"). The user requiring detailed information would be wise to start here for names of symbols and simple facts, but follow up with an authoritative subject-specific resource.Kristina L. Anderson, University of Alabama, kanderso@bama.ua.edu

JURIST. Access: http://jurist.law.pitt.edu.

Although subtitled "The Law Professors" Network," JURIST has a multitude of services to offer anyone from high school students to library patrons looking for the answer to a legal question. The site has won numerous awards for quality including Lycos Top 5\%, Infoseek Select Site, and a 5-Star rating from Legal.online. These awards are well warranted. JURIST is a comprehensive site in terms of content and its layout, design, and utility are outstanding.

On the front page of the site a search interface resides at the top, a navigation table at the left, and links for legal news and a database search are immediately noticeable. For a user looking for a current news story or a quick reference question, this layout is ideal.

News and access to datalsases are only the beginning for this site as the next category of links on the navigation table under "Start" is "Academic Pages." This set of links provides access to course syllabi, papers written for courses in legal education, and legal databases such as FindLaw, LEXIS-NEXIS (provided you have a password), and FedLiw. The "Presentations" link will take you to a list of tran-

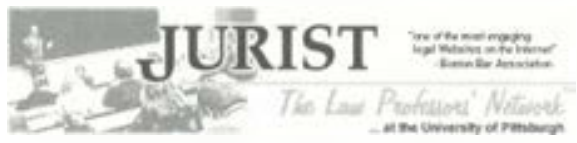

scripts or audiovideo recordings of recent conference presentations (the audiovideo recordings run on RealPlayer). "Examinations" has course exams, institutions' comprehensive examinations, and information on the bar. If you are interested in what's going on in legal education in other countries, the "JURIST Worldwide" link provides a list of professors pages from Ireland to China.

Sara Amato is electronic services and Web development librarian at Bowdoin College, e-mail:samato@bowdoin.edu 
Where JURIST really excels is in the "Services" set of pages. Books-on-Law offers reviews of recently published legal literature. The Media Center is a collection of all video and audio recordings throughout the JURIST site. The Reference Desk gathers directories, dictionaries, and links to major legal research sites with an online reference librarian, whose queries and answers are posted in a bulletin board format. Professors can find all the latest legal news at home and abroad through the Faculty Lounge, a bulletin board for announcements and requests, and JURISTChat-an "online interactive conversation and collaboration tool." Law students can find information from preparation for law school to job advertisements in the Student Lounge. It is difficult to imagine the information need not met by these services.

Navigation through the site is exceedingly easy as the navigation table is present on all pages except, of course, those not actually on the JURIST site but linked to as a resource. Only the few resources, such as the LEXIS-NEXIS database, are restricted to users with accounts. The vast majority of information collected by JURIST is free and open to everyone.

The JURIST site is well organized and exceptionally useful for anyone with questions about or an interest in the law, the legal profession, or legal education. Librarians will find it an excellent site for reference service or collection development in law or for just keeping up with current events.-Kinsten Tozer, Central Washington University, Tozerk@ taboma.curedu

Hispanic/Latino News Service. Access:

http://www.latinowww.com/.

The Hispanic/Latino News Service is a wellorganized clearinghouse and free abstracting service that points to Latino-related news articles published on the Web. In existence since May 1998, it has fast become one of the leadets in collecting and presenting current U.S. Latino news on the Internet. Webmaster Markos Alberto Moulitsas Zuniga, a third-year law student at Boston University School of Law, updates the site each weekday by browsing major online English-language news sites (such as the New York Times, Wasbington Post, and the L.A.Times) and a large number of regional online newspapers looking for U.S. Latino-oriented news stories to abstract and link.
In January 1999, a Spanish-language sec tion was introduced to the Web site, covering original Spanish-language news articles from online sources such as Miami's El Nuevo Herald (http://www.elherald.com/) and Chicago's La Raza (http://www.laraza.com/), an excellent online newspaper that covers many different U.S. Latino and Latin American groups. The Spanish-language section of the Hispanic/ Latino News Service is current but not updated daily, due to the fact that online U.S. Latino newspapers in Spanish tend to be weeklies. Thus, the site's current news in English covers the present week, while the Spanish current news section includes about three weeks of material. Archives are new to the site and date back only to mid-January 1999, with links no longer active; full bibliographic citations are also missing, making it necessary for scholarly users or librarians to do a few minutes of

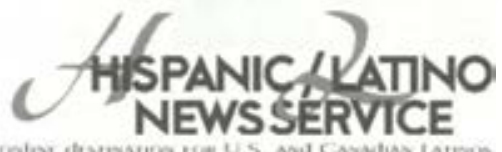

minor Web sleuthing to verify such things as dates, authors, and URLs.

Besides individual newspapers, other noteworthy electronic sources of U.S. Latino news include the Latino On-line News Network (http://www.latnn.com/), which provides its own original news articles in Spanish or English, and the subscription database Ethnic NewsWatch, the unsurpassed leader that provides original full-text (and mostly Spanish language) articles from over 20 Latino publications. Of these three sources, the Hispanic/ Latino News Service may have the broadest appeal for those lacking library access to Ethnic NewsWatch, and particularly for those who do not read Spanish. Overall, the currency and online accessibility of the Hispanic/Latino News Service make it at vital and attractive resource for all libraries interested in U.S. Latino viewpoints and issues.-Susan A. Vega Garcia, Iowa State University, savega@iastate.edu

\section{Reviewers sought}

If you are interested in becoming a reviewer for the "Internet Reviews" column please contact Sara Amato, e-mail: samato (1) bowdoin.edu for more information. 